\title{
Música y adolescencia: usos, funciones y consideraciones educativas
}

UT. Revista de Ciències de l'Educació

2015 núm. 2. Pag. 28-45

ISSN 1135-1438. EISSN 2385-4731 http://revistes.publicacionsurv.cat/index.php/ute

\author{
Salvador Oriola Requena ${ }^{a}$ y Josep Gustems Carnicer ${ }^{b}$ \\ Rebut: 25/07/2015 Acceptat: 14/11/2015
}

\section{Resumen}

Desde el nacimiento de la cultura adolescente a mediados del siglo $\mathrm{XX}$, los conceptos de adolescencia y música han formado un binomio inseparable, el cual ha ido afianzándose y variando en el tiempo gracias a los diferentes avances tecnológicos. Entre todos los avances tecnológicos destaca el uso de Internet a través de dispositivos móviles, pues ha permitido a los adolescentes tener acceso a cualquier tipo de música desde cualquier lugar. Todo esto ha repercutido en la forma de utilizar la música y en las funciones que ésta desempeña entre los jóvenes. Por esta razón, el presente artículo trata de ofrecer una visión psicosocial de los adolescentes actuales y de analizar cómo el uso y las funciones que desempeña la música en la vida de éstos han ido aumentando en diversidad y cantidad. Además ofreceremos diversas propuestas didácticas con el fin de sacar el máximo rendimiento educativo a un recurso tan efectivo y omnipresente en la vida adolecente como es la música.

Palabras clave: Adolescencia, educación musical, identidad, música

\begin{abstract}
From the origin of the teenager culture in the mid-twentieth century, adolescence and music concepts have formed an inseparable pair, which has been hold and changing due to the different technological advances. Among all the technological advances the use of Internet through mobile devices is the most remarkable, since it allows teens to access to any music from anywhere. All this has impacted on how young people use music and the functions it plays in their lives. Therefore, we have written this article to review how teenagers are today and we will analyze how the use
\end{abstract}

\footnotetext{
a Universitat de Barcelona

b Universitat de Barcelona
} 
and the roles that music plays in their lives have been increasing in variety and quantity. We will also offer various educational proposals in order to take advantage of music such an effective and ubiquitous resource in teenager lives.

Keywords: Adolescence, music education, identity, music

\section{Introducción}

La música es un elemento socio-cultural que ha estado presente en todas las sociedades conocidas desempeñando distintos roles, los cuales han ido variando a través del tiempo y las diferentes culturas. Todos estos cambios han sido abordados desde diferentes campos y posicionamientos teóricos derivados de la antropología, la sociología, la etnomusicología o la psicología de la música, entre otros (Nettl, 1956).

Hasta la primera mitad del siglo XX, tal y como afirma Cook (1998), la única música a la cual se tenía acceso era la música en vivo, ya fuese en una sala pública de conciertos, en un salón privado, en una fiesta al aire libre, en la iglesia, etc. Con el desarrollo tecnológico y la aparición de la música grabada y la radio, desde mediados del siglo $\mathrm{XX}$, tanto el acceso como el proceso de creación de cualquier tipo de música han ido cambiando de forma apresurada. Ésta es la razón por la cual en la actualidad, las oportunidades para acceder a la música se han ampliado y diversificado más que en cualquier otro momento de la historia. Todos estos cambios se han visto reflejados en las distintas funciones atribuidas a la música, que han aumentado en diversidad y en cantidad.

Uno de los primeros textos dedicados al análisis de los diferentes roles desempeñados por la música en el seno de cualquier sociedad fue The Anthropology of Music, del antropólogo A. Merriam (1964), el cual dedicó todo un capítulo a esclarecer el significado y la diferenciación entre los conceptos de usos y funciones, que, aunque son términos complementarios entre sí, muchas veces se intercambian y se confunden. Según Merriam, "usos" son las diferentes maneras cómo los miembros de una sociedad emplean la música en su día a día, ya sea tocando un instrumento, escuchándola o como complemento de otras actividades. En cambio, cuando se habla de "funciones", se requiere del análisis para conocer el porqué de los usos y sus propósitos, debiendo indagar para conocerlas, pues no son tan evidentes como lo son los usos. Así pues "los usos se refieren a la situación en la que la música es utilizada por las personas y las funciones tratan de esclarecer las razones por las cuales se hacen estos usos, concretamente conocer para qué sirve" (Merriam, 1964, p. 210).

A partir de esta distinción Merriam propuso diez tipos de funciones principales para la música que englobarían la mayoría de situaciones presentes a lo largo de la historia y de las culturas (Figura 1): 


\begin{tabular}{|c|c|}
\hline \multicolumn{2}{|r|}{ Funciones de la música } \\
\hline Expresión emocional & $\begin{array}{l}\text { La música sirve como medio para transmitir y evocar una gran } \\
\text { variedad de emociones en los oyentes, en los intérpretes y en los } \\
\text { creadores-compositores. }\end{array}$ \\
\hline Placer estético & $\begin{array}{l}\text { La música como obra de arte proporciona, por su significado y } \\
\text { valoración estética, el goce tanto de los creadores como de los } \\
\text { oyentes. Esta función está presente en las culturas occidentales } \\
\text { pero es más discutible que se dé en culturas no alfabetizadas. }\end{array}$ \\
\hline Entretenimiento & $\begin{array}{l}\text { La música es un elemento de entretenimiento en todas las } \\
\text { culturas. En las culturas occidentales suele entretener por sí } \\
\text { misma y en culturas no alfabetizadas, este entretenimiento suele } \\
\text { ser fruto de la combinación entre música y otras actividades. }\end{array}$ \\
\hline Comunicación & $\begin{array}{l}\text { La música sirve como vehículo de comunicación de algo, aunque } \\
\text { no quede claro de qué, de cómo o a quién. La música no es un } \\
\text { lenguaje universal sino que está conformado por los códigos de } \\
\text { la cultura a la cual pertenece. De todas las funciones, esta es la } \\
\text { función de la que más se desconoce. }\end{array}$ \\
\hline Representación simbólica & $\begin{array}{l}\text { La música se utiliza en algunas sociedades como representación } \\
\text { simbólica de otras ideas y comportamientos. }\end{array}$ \\
\hline Respuesta física & $\begin{array}{l}\text { La escucha y la producción de música provoca, en multitud de } \\
\text { ocasiones, respuestas físicas en las personas involucradas, a } \\
\text { pesar de que muchas de éstas estén delimitadas por } \\
\text { convenciones culturales. }\end{array}$ \\
\hline $\begin{array}{l}\text { Reforzar el cumplimiento } \\
\text { de normas sociales }\end{array}$ & $\begin{array}{l}\text { En un substancial número de culturas y grupos sociales se } \\
\text { utilizan canciones para instruir a sus miembros sobre cómo } \\
\text { comportarse, transmitiendo aquello que está bien o está mal. }\end{array}$ \\
\hline $\begin{array}{l}\text { Validar instituciones } \\
\text { sociales y rituales } \\
\text { religiosos }\end{array}$ & $\begin{array}{l}\text { En todas las culturas, las instituciones sociales y las religiones } \\
\text { usan canciones para reafirmar su vigencia y predicar sus } \\
\text { mensajes. }\end{array}$ \\
\hline $\begin{array}{l}\text { Contribuir a la estabilidad } \\
\text { y a la continuidad de la } \\
\text { cultura }\end{array}$ & $\begin{array}{l}\text { La música es un elemento presente en todas las sociedades } \\
\text { conocidas, desempeñando -como estamos viendo- un amplio } \\
\text { abanico de funciones, por ello forma parte de la cultura de cada } \\
\text { sociedad y contribuye a su continuidad. }\end{array}$ \\
\hline $\begin{array}{l}\text { Contribuir a la integración } \\
\text { social }\end{array}$ & $\begin{array}{l}\text { La música y las actividades que se combinan con ésta necesitan, } \\
\text { mayoritariamente, de una participación colectiva, la cual requiere } \\
\text { de cooperación y coordinación por parte de los miembros de } \\
\text { una sociedad. De esta forma, el uso de actividades musicales por } \\
\text { parte de los miembros de una sociedad, ayudará a la unión y la } \\
\text { integración de éstos. }\end{array}$ \\
\hline
\end{tabular}

Figura 1. Funciones de la música según A. Merriam (1964). 
Este intento de universalización sirvió como punto de referencia para el desarrollo posterior de un amplio corpus de investigaciones, relacionadas con el rol que ocupa la música en la vida diaria de las personas en cada cultura. Concretamente, en las últimas décadas del siglo XX proliferaron los desarrollos teóricos y las investigaciones desde diferentes disciplinas, entre las que destaca la psicología social de la música, que intentan demostrar y expandir todo este listado de funciones, contextualizándolas en emplazamientos y contextos culturales concretos, teniendo en cuenta la importancia, no solo de componentes cognitivos, sino también de componentes sociales y emocionales, factores indispensables para la validez ecológica de cualquier investigación sobre experiencias musicales cotidianas (Hargreaves, Marshall y North, 2003).

Basándonos en los posicionamientos anteriores, nos hemos planteado si la adolescencia tiene características específicas que adapten, modulen o agreguen algunas de estas funciones expuestas. Para ello se ha realizado una síntesis conceptual a partir de un análisis documental multidisciplinar enfocado en la psicología del desarrollo, la sociología de la educación y la educación musical que permita un acercamiento a los estudios y postulados teóricos centrados en la importancia de la música y las funciones que ésta desempeña en la vida de los adolescentes, protagonistas de nuestra investigación, con el objetivo de dar indicaciones de buenas prácticas que permitan un mejor aprovechamiento de esta materia en las etapas educativas propias de la adolescencia.

\section{La adolescencia, una edad controvertida}

A principios del siglo XX el psicólogo y pedagogo norteamericano S. Hall publicaba su obra Adolescence: its Psychology and its Relations to Physiology, Anthropology, Sociology, Sex, Crime, Religion and Education (1904), considerada por muchos como el primer tratado teórico sobre la juventud contemporánea. Era un compendio en el que se presentaba una visión romántica del concepto de "adolescencia", caracterizado por el Storm and Stress (tormenta y agitación), concepto equivalente al Sturm und Drang de los románticos alemanes, que describían la adolescencia como una etapa de polaridades y contradicciones constantes entre turbulencia $y$ melancolía, euforia y disforia, egoísmo y altruismo, soledad y ansia de amistad y vida grupal (González, 2001).

Hall definió la adolescencia como una etapa difícil de la vida que se extendería desde los 12-13 años hasta los 22-25. Muy en la línea del ilustrado franco-helvético Rousseau, hablaba de la adolescencia como un segundo nacimiento, una crisis, una transformación súbita y profunda provocada por la pubertad, una renovación total y dramática de la personalidad, una época de continuos cambios de humor, del despertar de la sexualidad, de repugnancia hacia la escuela y la familia, etc. Esta visión de crisis sería apoyada también por la teoría psicoanalítica de S. Freud (18561939), considerado junto con Hall como los precursores de la psicología de la 
adolescencia, que se centrarían en la sexualidad como el motor organizador de la personalidad, tanto en la infancia como en la adolescencia.

Desde entonces, han sido muchos los estudios y tratados destinados a describir y esclarecer las causas y detalles de esta etapa vital que, por así decirlo, no aparece diferenciada como tal hasta el s. XX. Entre las primeras teorías expuestas sobre la adolescencia y sus características encontramos los postulados de A. Gesell (18801961), que partiendo de las posturas biologistas de Hall y Freud, consideraría que los factores biológicos, en detrimento de los factores ambientales, determinarían el desarrollo de las personas (Gesell, Ames y Ilg, 1977). En un sentido parecido encontramos otra teoría relevante, la denominada teoría del establecimiento de la identidad, del psicoanalista E. Erikson (1902-1994). Este autor, discípulo de S. Freud e influenciado por las teorías antropológicas, establecería el desarrollo de la personalidad desde la niñez a la edad adulta a lo largo de ocho etapas. En cada una de estas etapas, el individuo tendría que superar una tarea psicosocial; en el caso de la adolescencia (quinta etapa para Erikson), la principal tarea sería el logro de la identidad, no sólo a nivel intrapersonal sino también interpersonal, es decir como miembro de una sociedad, de ahí la importancia de la influencia y la coherencia ideológica de la sociedad donde insertarse. Erikson afirma que la formación de la identidad tiene lugar gracias a las identificaciones que el adolescente acumula desde la niñez y utiliza el término "moratoria psicosocial" para referirse al periodo en que el adolescente realiza diferentes pruebas, retrocede, analiza y experimenta con diferentes roles sin tener que decidirse por ninguno, con el fin de prepararse para desempeñar las responsabilidades típicas de la vida adulta, especialmente las que tienen relación con el trabajo, la familia y la sociedad en general (Erikson, 1950/2008).

Desde la teoría del desarrollo cognitivo del psicólogo suizo J. Piaget (1896-1980), se propone el análisis de la génesis del conocimiento mediante estadios. Según Piaget al llegar a la adolescencia se producirán destacados cambios intelectuales, pues el individuo se integrará en la sociedad de los adultos, razón por la cual no se sentirá inferior sino que se situará al mismo nivel intelectual que estos últimos, alcanzando el denominado "pensamiento formal". Este tipo de pensamiento, que se iniciaría alrededor de los 11-12 años, supone la desvinculación de la lógica concreta de los objetos, y considera que el adolescente juega y utiliza ideas en lugar de limitarse a manipular objetos como hacía anteriormente, por ello será capaz de elaborar y comprender teorías y conceptos abstractos, así como razonar sobre la base de hipótesis (Piaget e Inhelder, 1966/1997). Es decir, el adolescente se aleja del mundo real, de lo directamente percibido, para adentrarse en el mundo de las hipótesis y sus comprobaciones, desarrollando de esta forma el pensamiento abstracto. Se utiliza un razonamiento hipotético-deductivo propio de la ciencia. También entra en juego el pensamiento proposicional y la deducción, mediante el cual el adolescente puede elaborar mentalmente las consecuencias lógicas que ocurrirían en cada una de las hipótesis si fueran ciertas y de esta forma asignar un grado de validez, que comprobará en la realidad antes de aceptarla como verdadera (Lara, 1996). 
A nivel educativo, la adolescencia es en la actualidad una etapa que se inicia estando los jóvenes inmersos en un proceso de escolaridad obligatoria, mientras que en las primeras décadas del s. XX, se entendía como la edad de iniciarse o aprender un oficio, colaborando con las tareas domésticas o aportando recursos económicos a las familias. Gracias a los cambios legislativos, laborales y sociales de las sociedades avanzadas, la adolescencia que hoy conocemos, encuadrada en la sociedad española de principios del siglo XXI, se caracteriza por ser una etapa vital situada entre la infancia y la adultez social, en la que los jóvenes se forman a través de la educación secundaria obligatoria desde los 12 a los 16 años y posteriormente el bachillerato o ciclos formativos, para acabar en la universidad o incorporarse al mundo laboral. Durante esta etapa los adolescentes, dependientes tanto legal como económicamente de sus progenitores, desarrollan su propia cultura y adquieren un estilo de vida caracterizado por unos valores y unos productos de consumo representativos de esta etapa, los cuales están relacionados con moda, música, revistas, videojuegos, etc.

Por lo que respecta a su duración total, actualmente la adolescencia se considera una etapa evolutiva más larga de lo que fue para las generaciones anteriores, el inicio y el final de este periodo se ha ido ensanchando progresivamente por razones diversas como la alimentación, la dilatación de la formación, el aumento de esperanza de vida, etc., de modo que su edad de comienzo es cada vez más temprana y su momento final se retrasa hasta una edad más avanzada (Tanner, 1986).

Otra característica digna de mención respecto a la imagen socio-emocional del adolescente, es la consideración de éste como persona problemática y conflictiva sumida por sus agitaciones emocionales. Esta concepción sobre la adolescencia basada en definiciones de tinte romántico ha perdurado durante muchas décadas, aunque en los últimos años se ha ido descartando, dejando al descubierto cierta falta de rigor científico. Hoy en día no se habla tanto de etapa tormentosa, sino más bien de un proceso vital dinámico por lo que respecta a los cambios externos e internos que se producen en la persona y que pueden comportar ciertos problemas de ajuste, al igual que ocurre con los cambios que se producen en otras etapas vitales, como la infancia o la vejez. Este ajuste no es homogéneo en todas las personas ya que influyen e interactúan una amplia diversidad de variables; por ello, el adolescente tendrá que adaptarse a las novedades que suponen los cambios físicos, intelectuales y socioemocionales propios de esta edad, fuertemente influenciados por el contexto en el que vive (Secadas y Serrano, 1981).

La autoestima es también uno de los conceptos que influyen en los cambios de comportamiento de los adolescentes. Rosenberg (1986) postula que al principio de la adolescencia es cuando las variaciones en la autoestima fluctuante son más intensas, cosa que mejorará y se estabilizará con el paso del tiempo gracias a un mayor conocimiento y aceptación del propio cuerpo y del yo real. El psicólogo norteamericano S. Harter (2003) distingue ocho factores que influyen en la imagen personal de los adolescentes: competencia académica, aceptación social, competencia deportiva, apariencia física, competencia laboral, atractivo romántico, 
comportamiento y amistad cercana. La apariencia física junto con las relaciones sociales que se dan entre el grupo de iguales aparecen como los factores más influyentes en la autoestima estable, especialmente en las adolescentes, las cuales enfatizan la apariencia como base de ésta y son más críticas con su aspecto físico, razón por la cual la autoestima de las mujeres es más baja que la de los hombres en la adolescencia.

El hecho de formar parte de un grupo de amigos o pares, además de influir en la concepción de la autoestima, también cumplirá una función social fundamental en el desarrollo socioemocional del adolescente, ya que de acuerdo con la psicóloga $\mathrm{G}$. Martínez (2003) la pertenencia a este tipo de grupos aportará:

- Soporte y comprensión. Los miembros del grupo se hallan viviendo circunstancias semejantes por eso pueden entender y aconsejar a sus compañeros y amigos.

- Sentido de pertenencia y estatus. El adolescente dentro del grupo adquiere unas actitudes y unas pautas de acción determinadas que puede aprovechar para su aplicación en diferentes contextos.

- Oportunidad de representar un papel y de percibir la propia competencia. Este punto está estrechamente relacionado con la moratoria social a la cual hemos hecho referencia anteriormente.

Como acabamos de comprobar, el estudio de la adolescencia desde una perspectiva socio-emocional ha estado tradicionalmente relacionada con el desarrollo de la identidad personal y con el aumento de la influencia del grupo de iguales en detrimento de la dependencia de los progenitores. En la actualidad, a estos rasgos hay que añadir otros, como la fuerte influencia de los cambios sociales y económicos que vive la sociedad, en el que las tecnologías de la información y la comunicación (TIC) ocupan un papel primordial.

Estas nuevas formas de relacionarse, de aprender, de ocio y el hecho de tener acceso a información ilimitada desde cualquier sitio y en cualquier momento a través del móvil, han sido estudiadas detalladamente por las industrias culturales, que proveen a los adolescentes de bienes y servicios tecnológicos especialmente producidos para ellos. Este nuevo mercado, conocido como teenage market, ha sido el responsable de moldear un nuevo tipo de adolescente caracterizado por el hecho de tener gustos y preferencias globalizadas (en música, moda, subculturas juveniles), acceso al conocimiento y dominio de las TIC superior al de sus mayores, mayor equiparación entre sexos, interacción entre la vida pública y la vida privada, preeminencia de la imagen y lo audiovisual... (Rubio, 2010).

En la era global en la que vivimos, los adolescentes adquieren una identidad cada vez más compleja fruto de la combinación de elementos culturales y elementos globales, es lo que Arnett (2008) denomina identidad bicultural o híbrida. Este tipo de identidad, además de ayudar a los jóvenes a percibir las diferencias existentes entre distintos contextos también les servirán como modelos identitarios, a partir de los 
cuales podrán escoger aquellos con los que más simpaticen y se aproximen a su forma de ser.

\section{Usos y funciones de la música en la adolescencia}

En el ámbito de los adolescentes, y especialmente en occidente, donde la adolescencia es más acusada, probablemente deberíamos cuestionarnos de modo general algunas de las funciones enunciadas por Merriam en nuestra introducción, dirigidas quizás más a un público adulto o infantil. Así pues, a nuestro parecer, reforzar el cumplimiento de normas sociales, probablemente sea propio de edades menores y de ámbitos educativos, así como validar las instituciones sociales y los rituales religiosos, se ve como algo alejado de la realidad habitual de los adolescentes occidentales, críticos y en general, apartados de las prácticas religiosas de sus comunidades de origen. El resto de funciones son claramente remarcables en estos colectivos, donde la música suele situarse como la actividad preferida, especialmente como pasatiempo.

En la segunda mitad del s. XX, y especialmente a partir del fenómeno "fan" basado en el consumo musical de los jóvenes y adolescentes, la preponderancia que tiene este colectivo frente a otros más estables y tradicionales es cada vez mayor. Los adolescentes americanos, por ejemplo, escuchan un promedio de 10.500 horas anuales, lo que representa una media de 3 horas diarias para chicos y 4 horas para chicas. La música es claramente la actividad favorita entre los jóvenes (94\%), tanto en chicos como chicas -por encima de los deportes-, lo cual repercute cuantitativamente en el negocio que mueve el consumo de la música de masas (North, Hargreaves y O'Neill, 2000). Uno de los motivos que contribuye a dicha afición y consumo es la incorporación de las nuevas tecnologías en la vida adolescente. Desde hace aproximadamente 20 años, el uso de dispositivos móviles ha permitido a sus usuarios acceder a todo tipo de música desde cualquier lugar y en cualquier momento, razón por la cual las funciones que desempeñaba la música hasta el momento han aumentado en cantidad y diversidad (Williams, 2007).

Por lo que respecta al plano educativo, a pesar de la inclusión desde hace décadas de la asignatura de música en el currículum de secundaria, no se ha conseguido marcar una diferencia, es decir, que la música ocupe un lugar significativo en la formación y el aprendizaje de los adolescentes. Ya en 1995 Ross constató que la música era una de las asignaturas menos populares en las escuelas de secundaria en Inglaterra, alegando que los intentos por modernizar el currículo de música en este país habían fracasado porque los profesores de música se habían estancado en lugar de reciclarse y adaptarse a los nuevos retos; porque los currículums estaban muy enfocados a la consecución de aspectos teóricos, dejando en un lugar secundario la creación y la interpretación de todo tipo de música, etc. Se detecta, pues que la música no puede ser enseñada como cualquier otra asignatura escolar convencional en estas edades, por ello deberían proponerse desde los institutos alternativas que se adapten a las preferencias y a las realidades sociales de los alumnos. Uno de los 
mayores problemas señalados es que existen grandes divergencias entre la música que los adolescentes escuchan en casa y la que escuchan en los centros educativos. La importancia capital de la música en la vida y la identidad de muchos adolescentes parece desarrollarse más fuera de las aulas que dentro de ellas: la música en la escuela está desligada de la importancia de la música referida a las necesidades de los adolescentes.

En el instituto, el adolescente empieza a reflexionar y descubrir que utilizando la música puede conseguir objetivos tan diversos como aislarse de su entorno, modificar su estado de ánimo, establecer y fortalecer relaciones interpersonales, etc., todos ellos objetivos muy significativos para esta etapa vital. Esta es una de las razones por las que la música preferida ocupará un lugar central en la vida de la gran mayoría de adolescentes.

Por lo tanto, entre los múltiples usos y funciones que desempeña la música en la vida de los adolescentes, podemos destacar:

- Expresión y regulación emocional. De acuerdo con Juslin y Sloboda (2010), la música evoca y despierta respuestas afectivas similares a las que se experimentan con cualquier otro tipo de emoción de carácter utilitario. Entre estas respuestas encontramos reacciones neurofisiológicas, reacciones comportamentales y reacciones cognitivas, como por ejemplo: escalofríos, tranquilidad, relajación, estímulo, etc., en resumen, puede servir como instrumento para autocontrolar el nivel de arousal (grado de activación neurofisiológica, motora y cognitiva que conllevan las respuestas emocionales). Además, como función integrante de la competencia emocional, también puede ayudar a intensificar las emociones cotidianas así como mejorar los estados de ánimo. La música puede ser útil para aliviar la tensión y el estrés (por ejemplo en épocas de exámenes, vacaciones, viajes, etc.), distraerles de las preocupaciones, como pasatiempo y para evitar el aburrimiento, situaciones propias y frecuentes en la adolescencia. Múltiples estudios llevados a cabo en países diferentes coinciden en señalar que la principal razón por la cual los adolescentes utilizan la música es la consecución de todos estos tipos de fines emocionales que acabamos de enumerar (Miranda y Claes, 2009; Saarikallio y Erkkilä, 2007).

- Memoria y evocación emocional. Relacionada con la función anterior, encontramos la música como medio articulador de la memoria emocional. Los adolescentes suelen vincular canciones a situaciones vividas, a las que atribuyen un significado especial, por ello cada vez que quieren rememorar dichas situaciones y sentir las emociones experimentadas, vuelven a escuchar la misma canción (Flores, 2008). El musicólogo británico S. Frith (1987) también atribuye a esta función el ser un recurso para la configuración de la memoria colectiva, es decir, que en multitud de ocasiones la música nos sirve como medio para organizar el sentido del tiempo e intensificar las experiencias vividas en grupo. Así mismo, la 
capacidad evocadora y creativa de la música está presente especialmente entre aquellos que tocan cualquier tipo de instrumento musical. Esto explicaría en parte el halo o carisma creativo en que se arropan los líderes de los grupos musicales de moda (North, Hargreaves y O'Neill, 2000).

Fuente de placer estético y entretenimiento. Éstas son dos funciones interrelacionadas, que a pesar de pertenecer claramente al dominio emocional, también forman parte del dominio cognitivo, pues disfrutar con la música está relacionado, de forma directa y sustantiva, con escuchar, valorar e interpretar música de cualquier estilo para poder reconocer y apreciar su valor estético o para que consiga entretener al oyente. En el terreno de la educación emocional, algunos autores, se refieren a las emociones producidas por la música como emociones estéticas (Bisquerra, 2009; Konečni, 2008). Por lo que respecta al entretenimiento y a la distracción, la música es una de las actividades favoritas de los adolescentes para pasar el tiempo y hacer frente al aburrimiento. Escuchar música ocupa en España el tercer lugar en actividades de ocio juveniles, por detrás de usar el ordenador y estar con amigos (Moreno y Rodríguez, 2012). En Estados Unidos e Inglaterra los adolescentes dedican entre dos y tres horas diarias a dicha actividad (Roberts, Henriksen y Foehr, 2004; Tarrant, North y Hargreaves, 2000). También cabe remarcar que muchas actividades de ocio y entretenimiento en esta etapa vital giran en torno a aspectos musicales, como puede ser cantar canciones de sus grupos favoritos, bailar en discotecas o tocar un instrumento. En general, las chicas presentan actitudes más positivas hacia la música que los chicos de todas las edades, de hecho, las adolescentes son más propensas a tocar instrumentos musicales que los chicos, y también asisten a más conciertos (North, Hargreaves y O’Neill, 2000).

- Acompañamiento de otras tareas. La cantidad de tiempo que se dedica a la música va en alza, debido al aumento del uso de dispositivos móviles como medio para la socialización a través de redes sociales y como instrumento multimedia, que permite al adolescente acompañar con música todas aquellas actividades que realiza con el ordenador portátil o con el móvil (Miranda, 2013). Esta es la razón por la cual la música, además de entretener, cumple la función de acompañar otras tareas, es decir se usa como sonido de fondo para evitar el silencio ambiental en actividades como caminar, estudiar, utilizar el ordenador o estar con amigos (Lonsdale y North, 2011).

- Establecer y consolidar relaciones interpersonales. Como estamos viendo, la música ocupa un lugar muy importante en la vida de los adolescentes, por ello un tema recurrente en muchas de sus conversaciones es hablar sobre canciones, sobre cantantes, sobre conciertos, etc. Todo esto forma parte, claramente, de la competencia social, puesto que se utiliza la música como nexo de unión entre el grupo de iguales y amigos. De acuerdo con Frith 
(1981), una de las primeras características a que hacen referencia los jóvenes para presentarse y describirse ante gente que no conocen, son sus preferencias musicales. En la actualidad esto se puede trasladar al perfil que cada uno tiene en redes sociales como Facebook, donde una de las informaciones más destacadas que aparecen, respecto a los datos que cada persona quiere mostrar para que se le conozca públicamente, son sus preferencias musicales. En la adolescencia, los gustos musicales sirven para darse a conocer y conocer mejor a los demás. Tener los mismos gustos musicales, significa mucho más que coincidir por lo que respecta a una valoración estética, es sinónimo de atracción social ya que se comparten puntos de vista, valores, creencias, formas de vestir, etc. (Selfhout, Branje, ter Bogt, y Meeus, 2009).

- Formación y consolidación de la identidad. Relacionada estrechamente con la función anterior y también dentro de la competencia social, encontramos que los gustos musicales de cada persona representan un componente más de su propia identidad. El adolescente se identifica con el género musical que le gusta, con su grupo favorito, con un cantante..., lo cual se manifiesta en muchas ocasiones a través de elementos extramusicales como puede ser la forma de vestir, su peinado, la ideología, el comportamiento, etc. De esta forma reafirman su personalidad y su identidad ya sea de tipo étnico, cultural, de género o colectiva (Rentfrow, 2012). La participación en muchas actividades musicales extra-curriculares puede incrementar su popularidad y estatus entre sus iguales.

Los adolescentes emplean la música como una guía de las características de los fans de determinados estilos y como medio para definir sus propias identidades, comunicando sus valores, actitudes y opiniones de los demás. Además los gustos musicales aparecen como un valor añadido de seducción y atracción ante el colectivo de iguales del otro sexo, tal y como demuestra un estudio realizado en Inglaterra, según el cual las chicas a quienes les gusta la música clásica, son percibidas como más atractivas por los chicos que aquellas que prefieren heavy metal (North, Hargreaves y O'Neill, 2000). Sobre las preferencias musicales, de acuerdo con Santos (2003), los adolescentes españoles se inclinan por el pop-rock internacional, seguido del pop-rock nacional, de hecho la adolescencia es considerada por muchos como la edad del pop, aunque aquellos que escuchan determinadas formas de música pop, tales como heavy metal o el rap, parece que sean más proclives a los comportamientos delincuentes que aquellos que escuchan otros estilos (Sharman y Dingle, 2015).

Las chicas informan que la música podría ser utilizada como un medio de regulación de las emociones, mientras que para los varones la música puede ser un medio para crear cierta impresión en los demás. La formación de la identidad y la gestión de las emociones son dos de las principales razones que los adolescentes estadounidenses alegan para escuchar música pop. 
Siguiendo la teoría de la Identidad Social (Tajfel, 1978), los individuos se perciben y se comportan más favorablemente hacia aquellos otros de quienes se percibe que comparten sus gustos musicales, de aquellos que no. El favoritismo intragrupo está motivado por la necesidad de incrementar la autoestima. Las preferencias musicales son uno de los posibles criterios para ser aceptado como miembro de un grupo, funcionando como "distintivo" que permite distinguir a quienes pertenecen a un grupo de quienes quedan fuera de él. El estar a la moda se consigue también compartiendo los gustos musicales de la mayoría, y esto correlaciona negativamente con los que tocan y escuchan música clásica, que les hace de alguna manera más independientes de las opiniones de los compañeros (North, Hargreaves y O'Neill, 2000).

- Lucha por la diferenciación respecto al grupo. Este es un movimiento pendular de defensa de la individualidad. Las preferencias musicales son compartidas por grupos de fans pero a su vez cada uno de ellos suele tener también algún tipo de preferencia algo distinta que el resto de compañeros: una canción en especial, un miembro del grupo que quizás pase desapercibido para las masas... Es una contradicción entre lo comercial y lo auténtico (Grossberg, 1992), que a lo largo de la adolescencia irá guiando su relación con el grupo más cercano y con el colectivo más general. La autenticidad de la música no es debida a la música propiamente sino a las personas que la hacen e interpretan (Moore, 2002). La capacidad de los cantantes de representarse como líderes de los colectivos, que hablan con sus pensamientos, encarnan experiencias míticas y representan la naturalidad y la autenticidad del colectivo, es algo que los adolescentes adoran (Gilbert y Pearson, 1999).

Respecto a la influencia que ejercen los gustos musicales de los padres sobre los hijos, cabe destacar que intervienen factores múltiples como la formación académica de los progenitores (especialmente del padre), el contexto, etc., pero se ha comprobado que sí que tienen una destacada influencia en el gusto por la música clásica de los hijos adolescentes y sobre su formación musical extraescolar. En general estos hijos suelen manifestar una menor ruptura respecto el ambiente familiar (Santos, 2003).

\section{Consideraciones educativas}

La música como recurso educativo forma parte de distintas competencias, entre las cuales destaca la competencia artística y las derivadas de las competencias cognitivas y emocionales. La música en España es una asignatura común integrada en el currículum de la educación secundaria obligatoria con el objetivo de contribuir a la consolidación de una serie de competencias básicas, las cuales están orientadas a la aplicación de los saberes adquiridos en distintos contextos. Por tanto la función educativa de la música tiene una doble función: la educación para la música, que 
consiste en el aprendizaje de elementos puramente musicales como puede ser tocar un instrumento, el lenguaje musical, la improvisación, etc.; y la educación a través de la música, que consiste en el uso de la música para desarrollar, además de la inteligencia musical, otro tipo de procesos cognitivos relacionados como pueden ser la creatividad, la imaginación, la capacidad de percepción o incluso, el lenguaje y las matemáticas (García, del Olmo y Gutiérrez-Rivas, 2014).

Las preferencias musicales de la etapa final de la adolescencia forjarán los gustos musicales de los futuros adultos de nuestra sociedad, sus costumbres culturales, su papel como consumidores y experimentadores de la música, y la orientación musical de las futuras generaciones de ciudadanos. A continuación apuntamos algunas consideraciones que hacen referencia al papel de la música en la adolescencia y que pueden servir de orientación a los educadores de estas etapas. Todas ellas van encaminadas a mejorar el papel y rendimiento de la música en la escolaridad de estas edades, con el fin de educar en lo posible el gusto musical de los adolescentes y desarrollar su capacidad crítica para hacer frente a la gran variedad de estilos musicales presentes en cualquier sociedad:

- Hoy en día la gran mayoría de música que escuchan los adolescentes se hace a través de los mass media dejando en un lugar residual la presencia de la música en directo. Para suplir este desequilibrio hace falta aumentar la asistencia a conciertos en vivo, para ello es recomendable conocer la oferta de conciertos didácticos, audiciones escolares, etc., que proponen auditorios y entidades culturales de las poblaciones donde vivimos.

- De todas las funciones que Merriam atribuyó a la música, los adolescentes conocen algunas de ellas -fruto de su práctica y experiencia musical-, aunque en general, no han reflexionado mucho al respecto. Para profundizar en este aspecto se proponen a continuación algunas actividades. Para la expresión emocional, planteamos que los alumnos escuchen música e intenten enunciar los contenidos emocionales de diferentes piezas, el estado de ánimo que el compositor ha intentado transmitir en cada una, la emoción que percibimos al escucharlas, etc. Como ejemplo se proponen especialmente aquellas obras no muy conocidas por los alumnos y que permiten una cierta ambigüedad en sus connotaciones, como la "marcha fúnebre para una marioneta" de Ch. Gounod, utilizada por Hitchcook como portada en sus series televisivas de misterio.

- Respecto al placer estético podemos proponer que cada alumno presente una pieza al grupo y de entre todas las piezas se escoja por votación la pieza que más les guste, como una especie de disco de oro, "Grammy"..., de este modo todo el grupo ejercitará la escucha activa con el fin de encontrar placer estético puro. Si los resultados no son homogéneos, se puede plantear hacerlo por sexos, pues los estilos preferidos seguramente diferirán entre chicos y chicas. 
- En referencia a la función de la música como entretenimiento, se propone hacer un listado de actividades que llevan siempre música de fondo, otras que pueden realizarse o no con música, y otras que nunca se realizan con música, para proponer cambios entre sí y experimentar sus consecuencias. Por ejemplo, estudiar con distintas músicas de fondo, realizar tareas domésticas con unas músicas u otras, etc. De esta forma el alumno reflexionará sobre la importancia del silencio y el abuso de la música en muchas situaciones diarias, lo cual puede ser una distracción en vez de una ayuda en el desempeño de algunas tareas.

- Por lo que respecta a la música como elemento de comunicación, se propone escuchar individualmente una obra y a continuación intentar explicar cuál es su argumento, motivación, posible título, etc. Para ello pueden utilizarse piezas programáticas -aquellas que a través de la música intentan describir una situación- sin que se explique el programa ni el argumento inicial, para ver el grado de coincidencia de cada cual. Un posible ejercicio sería escuchar "El aprendiz de Brujo" de P. Dukas y después de haber discutido sobre su posible trama argumental o temática, visualizar la versión que W. Disney hizo en su famoso film Fantasía.

La respuesta física que provoca la música es algo que puede experimentarse fácilmente mediante algún ejercicio de relajación dirigida. Para ello se medirá el ritmo cardíaco de cada cual con un reloj, se anotará y a continuación, en una postura estirada, se escuchará alguna pieza que ayude a la relajación -intensidades suaves, tempos lentos, etc.- como por ejemplo los primeros 7 minutos del Adagio de la $4^{a}$ sinfonía de Mahler. A continuación se tomará de nuevo la medida del pulso y se comparará con la primera medida. De esta forma, los alumnos comprobarán como la música puede ser un recurso para la relajación. Lo mismo podemos hacer en sentido contrario, con música estresante -intensidades fuertes, sonidos estridentes, etc.como por ejemplo, Death Metal, o la música de B. Hermann para la escena del acuchillamiento en la ducha de Psicosis.

- Hacer un listado de todas aquellas normas sociales, trucos, leyes, etc. que los alumnos aprendieron mediante una canción en el jardín de infancia, en la escuela, en campamentos, etc. Este listado les ayudará a reflexionar y ser conscientes de cómo la música ha sido utilizada desde pequeños en el cumplimiento de normas sociales. A partir de esta deducción se puede sugerir a los alumnos que improvisen y creen canciones propias con el fin de ayudarles a aprender y recordar reglas, conceptos o hábitos cotidianos.

- $\quad$ Acerca de la música como recurso para la integración social se propondrá que cada alumno aporte para toda la clase, una canción o danza de su lugar de procedencia. De esta forma todos los alumnos tienen la oportunidad de conocer y apreciar músicas de otras culturas, contribuyendo a un mejor conocimiento experienciado de las realidades sociales y culturales, y de este modo contribuir a la integración social de todos sus miembros. 
Respecto a la música como representación simbólica, validación de instituciones sociales y religiosas o estabilidad de la cultura, los adolescentes no suelen ser nada conocedores de dichos temas por su excesiva abstracción. No obstante, se propone que hagan un listado de unas 10 piezas musicales que crean indispensables en su fonoteca, posteriormente analizarán sus resultados para que sean conscientes de lo sesgado de sus preferencias y el alejamiento que puede darse respecto a la música tradicional de su cultura, las músicas que les han enseñado durante la educación, con la familia, etc. También se propone que valoren el conocimiento que cada uno posee acerca del himno nacional de su país, lo cual denotará su orientación simbólica respecto a su patria. Asimismo se propone que hagan un listado de himnos o canciones religiosas que conozcan y hayan practicado en celebraciones de esta índole para ver el grado de implicación o distanciamiento respecto a la experiencia religiosa.

- Sobre las implicaciones de la apariencia física en la identidad de los fans de un cantante, se propone comparar los rasgos generales de los alumnos con los rasgos físicos de los cantantes que más les gustan, para ver si hay elementos de coincidencia que indicarían cierta emulación vicaria en sus preferencias musicales, más allá de la música.

- Sobre la relación de sus gustos musicales con los de sus amigos y compañeros, se propone hacer un sociograma musical de una clase. Para ello debemos averiguar las preferencias musicales de cada alumno y el origen de sus gustos musicales. Asimismo se propone comparar este mapa conceptual con el mapa de afinidades intragrupo, para poder establecer correlaciones y liderazgos relacionados con el tema de las preferencias musicales dentro de la clase como modo de adquisición de estatus.

- Respecto a las identidades híbridas, sería interesante cotejar los resultados de 5 preferencias musicales con otras variables como el nivel sociocultural de la familia, la escuela primaria de procedencia, el país o cultura de origen, el sexo, etc, dado el carácter marcadamente multicultural de nuestros centros educativos. Con todo ello podríamos establecer y confirmar -si fuera el caso- algunas de las relaciones que se presentan en este artículo como el papel integrador de la música o la función de ésta como elemento de preservación de las raíces culturales de origen, la cual permita una mejor transición al mundo de la sociedad adulta.

- Hacer un análisis pormenorizado y una crítica sobre un concierto de un grupo o cantante famoso entre los adolescentes para establecer los parámetros en los que se basan los criterios musicales de éstos y entender cómo deberían presentarse alternativas a los repertorios habituales de la música en la Educación Secundaria Obligatoria (ESO).

- Elaborar un listado de piezas preferidas por los alumnos para conocer los gustos y las diferentes realidades sociales presentes en cada aula. A partir de 
las preferencias de los alumnos podremos crear y adaptar nuestro material didáctico, para explicar de forma más significativa, elementos de la asignatura de música como el lenguaje musical, las texturas, las formas y los géneros musicales, etc.

\section{Agradecimientos}

Este artículo está financiado por una ayuda a grupos de investigación ARCE de la Universidad de Barcelona, 2014, y forma parte de una investigación doctoral en la Universidad de Lleida.

\section{Referencias}

Arnett, J. J. (2008). Adolescencia y adultez emergente. México: Pearson.

Bisquerra, R. (2009). Psicopedagogía de las emociones. Madrid: Editorial Síntesis.

Cook, N. (1998). Music: A very short introduction. Oxford: Oxford University Press.

Erikson, E. (2008). Infancia y sociedad (N. Rosenblatt, Trad.). Buenos Aires: Hormé. (Trabajo original publicado en 1950).

Flores, S. (2008). Música y adolescencia. La música popular como herramienta en la educación musical. Tesis doctoral. Universidad Nacional de Educación a Distancia.

Frith, S. (1981). Sound Effects. Youth Leisure and the Politics of Rock ' $n$ ' Roll. Nueva York: Pantheon.

Frith, S. (1987). Toward an aesthetic of popular music. En R. Lepper y S. McClary (Eds.) Music and society (pp. 133-149). Cambridge: Cambridge University Press.

García, E., del Olmo, M. J. y Gutiérrez-Rivas, E. (2014). Educación musical y desarrollo cognitivo asociado. Música y Educación, (97), 28-41.

Gesell, A. Ames, L. B. e Ilg, F. (1977). Psicología evolutiva de 1 a 16 años (T. Reca, Trad.). Buenos Aires: Paidós.

Gilbert, J. y Pearson, E. (1999). Discographies: dance music, culture, and the politics of sound. Oxon: Routledge.

González, J. (2001). La construcción de las identidades de los jóvenes. Documentación Social, 124, 13-31.

Grossberg, L. (1992). We gotta get out of this place: popular conservatism and postmodern culture. Londres: Routledge.

Hall, G. S. (1904). Adolescence: its psychology and its relations to physiology, sociology, sex, crime, religion and education. Nueva York: Appleton Century Crofts. 
Hargreaves, D. J., Marshall, N. E. y North, A. C. (2003). Music education in the 21st century: A psychological perspective. British Journal of Music Education, 20 (2), $147-$ 163. doi: $10.1017 / \mathrm{S} 0265051703005357$

Harter, S. (2003). The development of self-representations during childhood and adolescence. En M. R. Leary y J. P. Tagney (Eds.), Handbook of self and identity (pp. 610-642). Nueva York: Guilford Press.

Juslin, P. y Sloboda, J. (2010). Handbook of Music and Emotion (ed. rev.). Oxford: Oxford University Press.

Konečni, V. J. (2008). Does Music Induce Emotion? A Theoretical and Methodological Analysis. Psychology of Aesthetics, Creativity, and the Arts, 2 (2), 115-129. doi: 10.1037/1931-3896.2.2.115

Lara, J. M. (1996). Adolescencia: cambios físicos y cognitivos. Ensayos, 11, 121-128.

Lonsdale, A. J. y North, A. C. (2011). Why do we listen to music? A uses and gratifications analysis. En British Journal of Psychology, $\mathrm{n}^{\circ}$ 102, pp. 108-134. doi: 10.1348/000712610X506831.

Martínez, G. (2003). Entre adolescentes: la importancia del grupo en esta etapa de la vida. En A. Perinat (Coord.), Los adolescentes en el siglo XXI (pp. 159-183). Barcelona: Editorial UOC.

Merriam, A. P. (1964). The Anthropology of Music. Evanston, Il: Northwestern University Press.

Miranda, D. y Claes, M. (2009). Music listening, coping, peer affiliation and depression in adolescence. Psychology of Music, 37, 215-233. doi: 10.1177/0305735608097245

Miranda, D. (2013). The role of music in adolescent development: much more than the same old song. International Journal of Adolescence and Youth, 18 (1), 5-22. doi: 10.1080/02673843.2011.650182

Moore, A. (2002). Authenticity and Authentication. Popular Music, 21(2), 209-223. doi: 10.1017/S0261143002002131

Moreno, A. y Rodríguez, E. (2012). Informe Juventud en España 2012. Madrid: Instituto de la Juventud.

Nettl, B. (1956). Music in primitive cultures. Cambridge, MA: Harvard University Press.

North, A. C., Hargreaves, D. J. y O'Neill, S. A. (2000). The importance of music to adolescents. British Journal of Educational Psychology, 70, 255-272. doi: 10.1348/000709900158083

Piaget, J. e Inhelder, B. (1997). Psicología del niño (A. Luís, Trad.). Madrid: Morata. (Trabajo original publicado en 1966). 
Rentfrow, P. J. (2012). The Role of Music in Everyday Life: Current Directions in the Social Psychology of Music. Social and Personality Psychology Compass, 6, 402-416. doi: 10.1111/j.1751-9004.2012.00434.x

Roberts, D. F., Henriksen, L. y Foehr, U. G. (2004). Adolescents and media. En R. M. Lerner y L. Steinberg (Eds.), Handbook of adolescent psychology, 2nd edition (pp. 487521). Hoboken, NJ: John Wiley \& Sons.

Rosenberg, M. (1986). Self-concept from middle childhood through adolescence. En J. Suls y A. Greenwald (Eds.), Psychological perspectives on the self, Vol. 3 (pp. 107-136). Hillsdale, NJ: Earlbaum.

Ross, M. (1995). What's wrong with school music?. British Journal of Music Education, $15,255-262$.

Rubio, A. (2010). Generación digital: patrones de consumo de Internet, cultura juvenil y cambio social. Revista de Estudios de juventud, 88, 201-221.

Saarikallio, A., y Erkkilä, J. (2007). The role of music in adolescents' mood regulation. Psychology of Music, 35, 88-109. doi: 10.1177/0305735607068889

Santos, F. J. (2003). Los gustos musicales de nuestros alumnos. Factores que determinan el gusto de la música clásica. Música y Educación, 55, 57-69.

Secadas, F. y Serrano, G. (1981). Psicología evolutiva: Edad 14 años. Barcelona: CEAC.

Selfhout, M., Branje, S., ter Bogt, T. y Meeus, W. (2009). The role of music preference in early adolescents' friendship formation and stability. Journal of Adolescence, 32, 95107. doi: 10.1016/j.adolescence.2007.11.004

Sharman, L. y Dingle, G. A. (2015). Extreme metal music and anger processing. Frontiers in Human Neuroscience, 9, 272. doi: 10.3389/fnhum.2015.00272

Tajfel, H. (1978). Differentiation between social groups. Londres: Academic Press.

Tanner, J. M. (1986). El hombre antes del hombre. México D. F.: Fondo de Cultura Económica.

Tarrant, M., North, A. C. y Hargreaves, D. J. (2000). English and American adolescents' reasons for listening to music. Psychology of Music, 28, 166-173. doi: $10.1177 / 0305735600282005$

Williams, A. (2007). Portable Music and Its Functions. Nueva York: Peter Lang Publishing. 